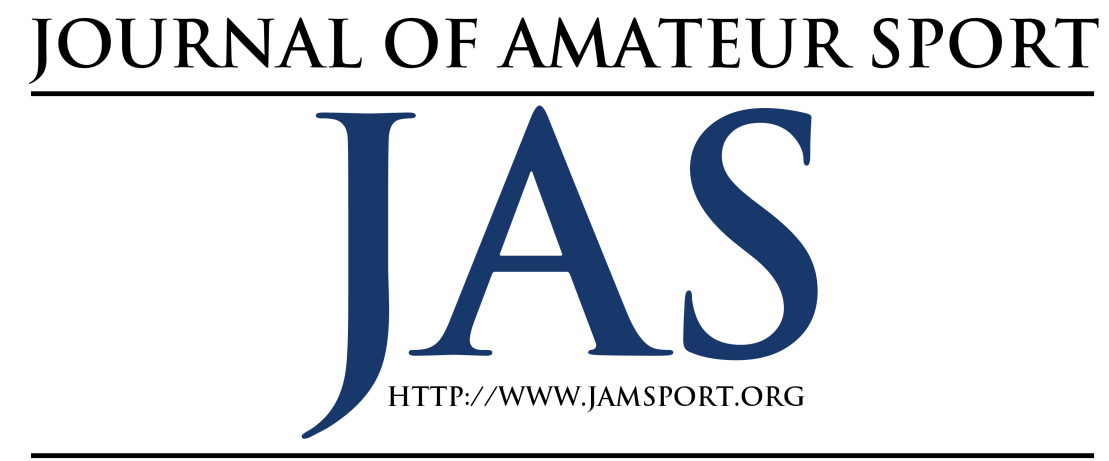

\title{
An Examination of the Relationship Between Team Cohesion and Individual Anxiety Among Recreational Soccer Players
}

\author{
Eungwang Oh Diane L. Gill \\ University of North Carolina at Greensboro
}

The purpose of this research was to examine the relationship between cohesiveness and competitive state anxiety and the contribution of cohesion to the prediction of competitive state anxiety among recreational soccer players. The research, which was conducted over two studies with recreational soccer players $(\mathrm{n}=47$ in Study $1 ; \mathrm{n}=$ 88 in Study 2), revealed negative relationships between sub-dimensions of cohesion and cognitive and somatic state anxiety. Further, individual attraction to the grouptask (ATG-T) contributed significantly to the prediction of state anxiety beyond the contribution of competitive trait anxiety. The findings suggest that recreational soccer participants with high ATG-T are likely to have low competitive state anxiety.

C

ohesion has been extensively studied as part of an attempt to develop an in-depth understanding of group dynamics in sport and exercise psychology (Brawley, Carron, \& Widmeyer, 1987; Carron, Brawley, \& Widmeyer, 2002; Carron, Widmeyer, \& Brawley, 1985). Carron, Shapcott and Burke (2007) noted that "cohesion is considered a distinguishing attribute of successful groups, whether it be in the domain of work, military, sport, or exercise" (p. 118). Research pertaining to group dynamics in sport has implications

for team performance enhancement and team success. For example, the effects of group size (Widmeyer, Brawley, \& Carron, 1990), group norms (Patterson, Carron, \& Loughead, 2005), and cohesion (Carron, Colman, Wheeler, \& Stevens, 2002) have been studied with a focus on team success. Other studies have recently examined intrapersonal or individual factors related to cohesion, which in turn affect overall group performance or individual group member's experience and behavior. Such individual factors associated with cohesion include 
positive mood (Terry et al., 2000), increased passion (Paradis, Martin, \& Carron, 2012) and satisfaction (Spink, Nickel, Wilson, \& Odnokon, 2005), to name a few.

It is noteworthy that cohesion, a group construct, is determined by individual perceptions of team environment in which they interact (Carron et al., 2002). Teams consist of individual members who are simultaneously influencing and influenced by one another. Furthermore, the team environment, which is developed by individuals, also affects individual cognition, feelings, and behavior. Bosselut, Heuzé, Eys, and Bouthier (2010) pointed out that the team environment impacts team members' psychological states. They reported that high group cohesion, particularly group integration-task (GI-T) could lead to a facilitative interpretation of cognitive anxiety. Beauchamp, Bray, Eys, \& Carron (2003) found that individual perception of role ambiguity and responsibilities within the team environment was associated with competitive A-state. Such findings imply that positive group factors including team cohesion can positively influence individual affective states during competition. Therefore, it is important to continue investigating intrapersonal or individual factors associated with cohesion, especially when the focus is on the individual experience in team sport, rather than team success.

In the most accepted definition, cohesion is described as "a dynamic process which is reflected in the tendency for a group to stick together and remain united in the pursuit of its instrumental objectives and/or for the satisfaction of member affective needs" (Carron, Brawley, \& Widmeyer, 1998, p. 213). This definition is based on a multifaceted conceptual model proposed by Carron et al. (1985). Carron et al. (1985) not only took into consideration the group, but also the individual aspect of cohesion. Further, the instrumental (task) and the interpersonal (social) aspects were included in the cohesion model.

Subsequently, four dimensions are identified in Carron et al.'s (1985) cohesion model: Group Integration-Task (GI-T), Group Integration-Social (GI-S), Individual Attractions to the Group-Task (ATG-T), and Individual Attractions to the GroupSocial (ATG-S). It should be noted that each component represents the different conceptual construct of cohesion.

- Group integration-task (GI-T): individual perception about the unity of team as a whole around task aspects (e.g., taking responsibility when failing to achieve the performance goals in the team).

- Group integration-social (GI-S): individual perception about the unity of team as a whole around social aspects (e.g., building up fellowship away from sport).

- Individual attractions to the grouptask (ATG-T): individual perception about personal interest in the group 
task. (e.g., liking performance related aspects of the team).

- Individual attractions to the groupsocial (ATG-S): individual perception about personal interest in social interaction with the group members (e.g., liking social interaction in the team). (See Carron et al. (2002) for more details)

Anxiety during competition is commonly experienced by athletes at all levels (c.f., Defrancesco \& Burke, 1997; Landers \& Arent, 2010). Accordingly, many researchers and professionals in sport and exercise sciences have paid special attention to precompetition anxiety in research and practice, enabling continual developments of measurements models along with empirical research (Hanton, Neil, \& Mellalieu, 2008). An extensive line of research on competitive anxiety began with Martens' (1977) early work and continues to expand in several directions. Martens drew on the psychology research of Spielberger (1966) and others who differentiate trait and state anxiety. Trait anxiety (A-trait) is a personality disposition, or tendency to become anxious in threatening situations. State anxiety (A-state) is an emotion, referring to the immediate feelings of anxiety. Martens described competitive trait anxiety (A-trait) as the tendency to perceive competitive situations as threatening, and respond with state anxiety. Martens (1977) developed a sport-specific measure of competitive A-trait, the Sport Competition Anxiety Test (SCAT). Considerable research supports that the SCAT has strong psychometric properties, and more importantly that competitive A-trait predicts competitive A-state in competition better than more general trait anxiety measures (Martens, 1977; Martens, Vealey \& Burton, 1990).

State anxiety is one of the most studied emotions in psychology, as well as in sport, and much of the research highlights the multidimensional nature of state anxiety with cognitive and somatic components. Similarly, competitive A-state is a multidimensional construct that involves immediate responses in competitive evaluation situations (Martens et al., 1990). Martens et al. (1990) developed the Competitive State Anxiety Inventory (CSAI) which assesses cognitive (cognitive A-state) and somatic (somatic A-state) state anxiety. Cognitive A-state is described as worry about negative outcomes while somatic Astate refers to physiological arousal in response to evaluative situations.

Both the SCAT and CSAI-2 are widely used in sport psychology research, and considerable research confirms that competitive A-trait (SCAT) predicts competitive A-state (e.g., Gill \& Martens, 1977; Martens et al., 1990). Weinberg and Gould (2014) indicated that competitive Atrait has a direct relationship to competitive A-state. Smith, Smoll and Wiechman (1998) concluded that in competitive sport situations, competitive A-trait is the primary predictor of competitive A-state. 
Research suggests that excessive anxiety experienced by sport participants has a variety of negative psychological and behavioral correlates, including loss of confidence, burnout, and possibly dropout from sports (Gould, Petlichkoff, \& Weinberg, 1984; Martens et al., 1990; Weinberg \& Gould, 2014). However, research also shows that group cohesion provides psychological benefits for athletes. The unity in groups has a positive influence while social exclusion from groups is associated with negative feeling states (Baumeister \& Leary, 1995). Cohesion is also associated with improved mood (Lowther \& Lane, 2002), and has a negative relationship with depression and tension (Terry et al., 2000). Prapavessis and Carron (1996) specifically suggested that cohesion may reduce individual competitive A-state. The notion that decreased levels of competitive A-state benefit athletes in various ways has been well-supported by a substantial body of research (Martens et al., 1990). Therefore, cohesive groups can foster cognitive, psychological, and somatic benefits in sport participants.

Previous research examining the relationship between cohesion and competitive A-state provides groundwork for the present study. Prapavessis and Carron (1996) recruited 110 athletes in rugby, basketball, hockey and soccer to examine the interrelationship between cohesion and competitive A-state. Their results showed that ATG-T and competitive A-state were negatively related, and that highly task-cohesive team members tend to perceive less cognitive A-state. Eys, Hardy, Carron, and Beauchamp (2003) further examined the relationship using the CSAI2d (Competitive State Anxiety Inventory 2 with a direction scale, Jones \& Swain, 1992) and reported that highly task-cohesive athletes positively interpreted their competitive A-state. Specifically, task cohesion distinguished athletes with positive perceptions of their competitive A-state from athletes with negative interpretations of their competitive A-state.

Research on the relationship between cohesion and competitive anxiety is limited and focused on elite competitive athletes (e.g., national or international level), or mixed samples at various competitive levels (e.g., intercollegiate, club, or competitive school level). Issues related to cohesion and anxiety are just as relevant to the larger number of participants in recreational sport competition. Group dynamics between elite and recreational athletes are likely to differ because of different group norms, rules, and roles across different skill levels. In fact, Carron et al. (2002) suggested that recreational sport teams and elite sport teams may have different group attributes. In their meta-analysis, it was found that the degree to which cohesion is related to performance differed across levels of competition. Therefore, individual affective states in relation to cohesion from a variety of competitive levels need further examination. In addition, sport takes place in more recreational environments than 
highly competitive settings (Widmeyer, Brawley \& Carron, 1990). At the recreational sport level, positive individual experiences (e.g., enjoyment, lower anxiety) are as important as group performance (Widmeyer et al., 1990). However, the importance of individual affective states in the recreational sport setting has been overlooked and the relationship between team cohesion and individual anxiety among recreational athletes has remained unexplored. Cohesion in recreational sport teams may be a way to enhance positive individual experiences. Participants' positive experiences in recreational team sports, such as less pressure and improved feeling states, can promote adherence and longterm physical activity. Spink and Carron (1993) found that cohesion is closely associated with individual adherence to sports. Exercise participants in the 8-week team building intervention groups had fewer dropout rate and late arrivals compared to the control groups. Particularly, ATG-T was reported to be the most relevant dimension for team building. It was revealed that individual adherence behavior can be influenced by individual perceptions of cohesion within the group. Therefore, an examination of the relationship between cohesion and competitive anxiety at the recreational level has implications for sport adherence behavior.

The purpose of this research was to examine the relationship of team cohesion with competitive A-state experienced by recreational sport participants. The present study extends previous studies on the relationship between multi-constructs of cohesion and multi-dimensions of competitive A-state to the recreational sport level. Also, no previous research has included competitive A-trait along with cohesion in relation to competitive A-state. Given that competitive A-trait is a wellestablished major predictor of competitive A-state, it should be a strong predictor in the current study. In the present study, the additional contribution of cohesion over and above the expected contribution of competitive A-trait to the prediction of competitive A-state was examined.

To address the purpose, the relationship of each dimension (Individual Attraction to the Group-Task, ATG-T; Individual Attraction to the Group-Social, ATG-S; Group Integration-Task GI-T; Group Integration-Social, GI-S) of the Group Environment Questionnaire (GEQ; Carron et al., 1985) to each component (cognitive A-state and somatic A-state) of the CSAI-2 was examined. Based on findings from previous research that demonstrated significant associations between task cohesion and competitive anxiety (Eys et al., 2003; Prapavessis \& Carron, 1996), it was hypothesized that individual attraction to the group-task (ATG-T) and group integration-task (GI-T) would be negatively related to cognitive A-state and somatic Astate. As previous research suggested that the strongest predictor of competitive Astate during competition situations was competitive A-trait, it was hypothesized that 
competitive A-trait would significantly predict competitive A-state and that individual attraction to the group-task (ATG-T) and group integration-task (GI-T) would contribute to the prediction of competitive A-state above and beyond competitive A-trait.

\section{Methods}

This research was conducted in two separate studies, both addressing the same research questions with recreational soccer players. Because both studies address the same research questions, using the same measures and similar procedures, methods and results are presented together with any specific differences noted. It is important to note that the two studies were conducted at different periods of time. Also, the characteristics of competition and participants were different; therefore, the two studies were analyzed separately. Power analysis was carried out based on data from the first study, and indicated that a sample size of 80 was adequate for the second study.

\section{Participants and Procedure}

In both studies, the competition was recreational or non-elite. Only those adults (age 18 and over) who participated in amateur local leagues/competitions mainly for recreation, without formal contract or compensation were recruited for this research. Non-elite or recreational soccer participants in this research were participating in local competition for recreational purposes, without being paid to play and stay on the team. Managers and competition organizers from the targeted amateur soccer leagues were contacted to request their cooperation for the present study. After obtaining approval from the Institutional Review Board (IRB), the primary researcher separately met the three competition organizers (one for Study 1, two for Study 2) and two managers (Study 2). Potential participants for Study 1 (approximately 110) were contacted in person and potential participants for Study 2 (approximately 340) were contacted at a team meeting or competition and later via email.

Study 1. Participants were recruited from a soccer tournament of the Korean American sport competition. This sport competition is popular among Korean immigrants residing in the U.S. and most teams practice months for this competition. Many recreational athletes stay on the same team across several seasons. This is reflected by years spent on the same team $(M=4.68$; $S D=4.06)$. Eight teams participating in the competition were met by the primary researcher. They were asked to voluntarily participate, and six of the eight teams participated. Participants completed questionnaires that included a demographic and soccer background questionnaire, the GEQ, the CSAI-2, and the SCAT immediately before competition. Of the 55 recreational athletes who agreed to participate, seven did not complete all of the questionnaires and one was excluded due to 
lack of English fluency (i.e., less than 4 [good] on a 6-point Likert scale [from poor to excellent] for those whose primary language is not English) and therefore were not included in subsequent analyses.

The 47 participants were all male soccer players, including two Caucasians (4\%), one African American (2\%), and 44 Asians (94\%; dominantly Koreans) regarding race/ethnicity. Because it was assumed that there would be participants whose primary language was not English, primary language and English fluency were examined. It was found that 22 participants used English as their primary language, 23 participants used Korean and two participants used other languages as their primary language. All scored at least 4 (good) on the 6-point rating of English fluency except for the one who was dropped from subsequent analyses.

Their ages ranged from 18 to 51 years old $(M=27.26 ; S D=7.57)$. Years of experience ranged from two to 40 years ( $M$ $=13.68 ; S D=8.10)$, while years on the current team ranged from 0 to 16 years $(M$ 4.68; $S D=4.06$ ). On average, the perceived individual soccer skill was 4.11, and their mean team skill level was 4.32 on a 6-point Likert scale. Their perceived importance of the competition was 4.51 on a 6-point Likert scale with higher scores reflecting higher skills and importance.

Study 2. Participants in community recreational soccer leagues were recruited for Study 2. Most of the recreational athletes stay on the same team from season to season, as reflected in the years on their team $(M=3.8 ; S D=4.05)$. The same teams participate in the same local recreational soccer leagues across seasons. All recreational athletes who agreed to participate were sent an e-mail that included an explanation of the study, informed consent, and a hyperlink to an electronic survey (Qualtrics). The survey included the same measures as in Study 1 - a demographic and soccer background questionnaire, the GEQ, the CSAI-2, and the SCAT. Reminder e-mails were sent twice to those who did not respond to the initial e-mail. It was emphasized that participation in the study was voluntary and confidential. Of the 114 recreational athletes who completed the online survey, 26 were excluded due to the incomplete surveys. Therefore, 88 participants were used for further analyses in Study 2.

The 88 participants in Study 2 were 79 male (89.8\%) and nine female (10.2\%) recreational soccer players who participated in local amateur soccer leagues. The leagues were co-ed with no minimum number of female players, but the large majority was male participants. Thus, the sample is representative of the leagues. A majority (68) of the participants identified as White/European (77.3\%) and 11 others as Asian (12.5\%), four as Black or African American (4.5\%), four as Hispanic/Latino (3.4\%), one as American Indian or Alaska Native $(1.1 \%)$ and one other (1.1\%). The average age of participants was 36.2 years old $(S D=11.78$; range $=20-66)$. 
The average years of experience was $22.9(S D=12.17$; range $=1-60)$, while years on the current team was $3.8(S D=$ 4.05; range $=0-22)$. On average, the perceived individual soccer skill was 4.0, and their mean team skill level was 3.8 on a 5 point Likert scale. Their perceived importance of the competition was 3.9 on a 5-point Likert scale with higher scores indicating higher skills and importance. In terms of team practice other than scheduled league matches, $72.7 \%$ answered 'never' and $14.8 \%$ answered 'less than once a week' while $12.5 \%$ indicated that they practice once or more than once a week. Concerning the most important reason to participate on the current soccer team, more than half of the participants $(54.5 \%)$ answered 'to have fun.' The other reasons were 'for the competition' (19.3\%), 'to socialize with others' (10.2\%), and 'to improve soccer skills and move to higher levels' (3.4\%) as their reason to participate. Interestingly, several participants chose other (12.5\%), reporting that fitness or combination of fitness, fun and socialization was the reason to participate on the current soccer team.

\section{Measures}

Demographic and soccer

background questionnaire. Demographic information on participants was collected using the demographic questionnaire. Demographic items included gender, age, and race/ethnicity. The soccer-related items included years of experience, years on the current team, perceived skill level, and importance of the competition for the current team. Frequency of practice for the current team and the most important reason for participating on the current team were asked as well. Demographic and soccerrelated information were used to better understand the individuals and teams in the present study.

\section{Group Environment Questionnaire.} Cohesion was assessed with the Group Environment Questionnaire (GEQ; Carron et al., 1985). The GEQ consists of four dimensions of cohesion. Five items assess Group Integration-Task (GI-T; e.g., "Our team is united in trying to reach its goals for performance"), four items assess Group Integration-Social (GI-S; e.g., "Members of our team do not stick together outside of practices or games"), four items assess Individual Attractions to the Group-Task (ATG-T; e.g., "I do not like the style of play on this team"), and five items assess Individual Attractions to the Group-Social (ATG-S; e.g., "I do not enjoy being a part of the social activities of this team"). Participants marked a 9-point Likert scale anchored at the extremes by strongly agree (9) and strongly disagree (1). In this measure, higher scores indicate higher perceptions of cohesion. Previous research has supported its validity and internal consistency (Brawley et al., 1987; Carron et al., 2002; Carron, Widmeyer, \& Brawley, 1985). Reliability for all measures in the current study is included in the results section. 
Sport Competition Anxiety Test. Competitive trait anxiety was assessed with the Sport Competition Anxiety Test (SCAT; Martens, 1977). The SCAT was developed to measure the predisposition to become anxious in sport competition. The SCAT includes 10 items with three responses (hardly ever, sometimes, or often) that indicate how respondents usually feel when in sport competitions (e.g., "When I compete, I worry about making mistakes"). The possible range of scores is from a low of 10 to a high of 30 , which reflects the highest competitive A-trait. Research supports the concurrent, predictive, and construct validity of the SCAT as a measure of competitive A-trait (Martens, 1977;

Ostrow \& Ziegler, 1978).

Competitive State Anxiety Inventory2. The Competitive State Anxiety Inventory-2 (CSAI-2; Martens et al., 1990) was used to assess competitive state anxiety levels (how participants feel at the moment). The CSAI-2 consists of three dimensions: cognitive anxiety, which measures conscious awareness of unpleasant feelings about oneself or the situation, somatic anxiety, which measures awareness of bodily symptoms of the autonomic nervous system, and self-confidence, which measures the degree of certainty that athletes feel about their ability to be successful. The CSAI-2 has 27 items presented on a four-point scale anchored at extremes by not at all (1) and extremely (4). Higher scores represent higher levels of cognitive anxiety (e.g., "I am concerned about losing"), somatic anxiety (e.g., "I feel my stomach sinking"), and self-confidence (e.g., "I'm confident about performing well'). Previous research supports validity and reliability through correlations between the CSAI-2 subcomponents and other anxiety measures (Martens et al., 1990). In the present study, self-confidence was not used as this scale is not considered a dimension of A-state.

Role play scenario. Study 1 participants completed measures prior to competition, but for Study 2, participants completed a role play scenario that asked them to think about a situation when they are about to compete in the competition with their teams. The role play scenario was used due to the unique features of recreational sport teams. Many participants come to the competition individually, often at the last minute, which hinders survey administration before competition.

A role play scenario has often been implemented in a variety of fields including sport and exercise psychology to assess emotion appraisal by recalling personal experience or relating it to a hypothetical scenario (e.g., Jones \& Uphill, 2004; Levine, 1996; Ntoumanis \& Biddle, 2000). Some researchers have used scenarios in competitive A-state research (Hanin, 2007; Levine, 1996).

In the scenario, a brief description of a soccer competition followed by specific instructions was provided to help the participants visualize their most recent important game and elicit thoughts and 
feelings in that situation at that moment. Participants were asked to imagine how they would react in that specific situation. This hypothetical scenario was designed according to instructions used by Ntoumanis and Biddle (2000) and Jones and Uphill (2004). Then, participants were asked to complete the CSAI-2 based on their feelings in the scenario. The GEQ and the SCAT were completed first, and then the CSAI-2 was completed with the role play scenario.

\section{Results}

Results are presented for both studies starting with reliabilities and descriptive analysis results followed by correlation and regression analyses.

\section{Reliabilities}

Initially, internal consistency was examined for all scales (see Table 1). All internal consistency values were acceptable except for ATG-S (Study 1, $\alpha=.37$; Study $2, \alpha=.32$ ) that showed substantially low internal consistency. Also, GI-S had a low reliability in Study $1(\alpha=.59)$, but acceptable reliability in Study $2(\alpha=.67)$. Thus, ATG-S was eliminated from regression analyses in both studies. The GI$S$ was retained, but the marginal reliabilities suggest caution in interpretation. All other internal consistency values were similar in both studies.

\section{Correlations}

Pearson's correlation analysis was conducted first to examine relationships of sub-dimensions of the GEQ with subcomponents of the CSAI-2 (cognitive Astate and somatic A-state) and competitive A-trait. The results of correlation analyses are presented in Table 2. As expected, GEQ dimensions were negatively correlated with cognitive and somatic A-state in both studies, indicating that higher scores on cohesion dimensions were associated with lower levels of cognitive and somatic Astate. However, in Study 1, only somatic Astate was significantly related to ATG-T, while task-oriented cohesion (ATG-T and GI-T) was significantly correlated with both cognitive and somatic A-state in Study 2 (see Table 2).

\section{Stepwise Multiple Regression}

In each study, two separate stepwise multiple regression analyses were conducted to examine the contribution of the cohesion sub-components in predicting cognitive and somatic A-state; cognitive and somatic Astate were the criterion variables, while the three GEQ sub-components (ATG-T, GI-T and GI-S) were entered in the multiple regression model as the predictor variables.

In Study 1, no significant multiple regression was found because no correlations were high enough to enter into the equation predicting cognitive A-state. In Study 2 , a significant result was found, $R^{2}=$ $.12, F(1,86)=12.12, p<.001$, with the multiple correlation coefficient of .35 and approximately $12 \%$ of the variance in 
cognitive A-state accounted for by ATG-T. ATG-T was the only predictor that accounted for a significant amount of variance in cognitive A-state, indicating that recreational soccer players with higher ATG-T have lower cognitive A-state.

The stepwise multiple regression model with somatic A-state as the criterion variable produced similar results in both studies; $\mathrm{R}^{2}$ $=.13, F(1,45)=6.91, p<.05$ in Study 1 and $R^{2}=.08, F(1,86)=7.81, p<.01$ in Study 2 . The results of the analysis indicated that the multiple correlation coefficient was .37 in Study 1 and .29 in Study 2, respectively reflecting approximately $13 \%$ and $8 \%$ of the variance in somatic A-state. In both studies, only ATG-T had a unique contribution to the prediction of somatic Astate.

\section{Hierarchical Multiple Regression}

Subsequently, two sets of hierarchical multiple regression analyses in each study were carried out with competitive A-state as the dependent variable to test the hypothesis that the GEQ sub-components add to the prediction of cognitive and somatic A-state beyond the contribution of competitive A-trait. Competitive A-trait was the first variable entered, followed by the GEQ subcomponents with the stepwise method, based on theory and previous research.

The hierarchical multiple regression models with cognitive A-state as the dependent variable first indicated that competitive A-trait contributed significantly to the regression model in both studies; $R^{2}$ $=.11, F(1,45)=5.28, p<.05$ in Study 1 and $R^{2}=.37, F(1,86)=51.05, p<.001$ in Study 2. In the second step, the only significant predictor of cognitive A-state was ATG-T in both studies; $\Delta \mathrm{R}^{2}=.10, \beta=$ $.31, t=-2.29, F$ change $(1,44)=5.25, p<$ .05 in Study 1, and $\Delta \mathrm{R}^{2}=.06, \beta=-.26, t=-$ $3.09, F$ change $(1,85)=9.57, p<.005$ in Study 2. ATG-T explained an additional $10 \%$ and $6 \%$ of variation in cognitive Astate beyond the contribution of competitive A-trait. That is, the prediction of cognitive A-state was significantly improved by adding ATG-T beyond the contribution of competitive A-trait.

The second set of hierarchical multiple regression models with somatic A-state as the dependent variable also showed that competitive A-trait contributed significantly to the regression model in both studies; $\mathrm{R}^{2}$ $=.17, F(1,45)=9.14, p<.005$ in Study 1 and $R^{2}=.59, F(1,86)=121.72, p<.001$ in Study 2. As predicted, after controlling for competitive A-trait, ATG-T made a significant contribution to predicting somatic A-state in both studies; $\Delta \mathrm{R}^{2}=.16, \beta$ $=-.41, t=-3.29, F$ change $(1,44)=10.82, p$ $<.005$ in Study 1 , and $\Delta \mathrm{R}^{2}=.03, \beta=-.16, t$ $=-2.39, F$ change $(1,85)=5.71, p<.05$ in Study 2. No other subcomponent of GEQ contributed significantly to the prediction of somatic A-state (See Table 3 and 4). 


\section{Discussion}

The aim of the present study was to determine the relationship between cohesion and competitive A-state among amateur soccer players. Specifically, the contribution of cohesion to the prediction of competitive A-state was examined. In addition, the relationship between cohesion and competitive A-state was investigated, while also including competitive A-trait as a predictor. It was hypothesized that ATG-T and GI-T would be negatively associated with cognitive and somatic A-state.

Pearson's correlation analysis indicated that the three cohesion variables (ATG-T, GI-T, and GI-S) were negatively related to cognitive and somatic A-state in both studies, with ATG-T demonstrating the strongest relationships with both somatic Astate and cognitive A-state. The findings suggest that when task cohesion (ATG-T, GI-T) is high in sport teams, team members may have low pre-competition anxiety. Prapavessis and Carron (1996) and Eys et al. (2003) showed similar correlations between cohesion variables and competitive A-state variables.

It is meaningful to note that most participants $(87.5 \%)$ in the second study reported that they never practice or practice less than once a week as a team. Only $12.5 \%$ of the participants reported that they practice more than once a week. This comes as no surprise in that more than half of the participants (54.5\%) indicated that their primary reason to play for their current team was to have fun. The other reasons to participate on their current team were the competition, social interaction, fitness, and combinations of these. Only 3.4\% of participants reported that their main reason to play for their team is to improve their soccer skills and move to higher levels.

Stepwise multiple regression results provide partial support for the hypothesis that ATG-T and GI-T predict competitive A-state. ATG-T accounted for significant variance in cognitive (approximately 12\%) and somatic A-state (approximately 8\%). However, GI-T was not a significant predictor of competitive A-state. The findings were consistent with Prapavessis and Carron's (1996) finding that ATG-T and cognitive A-state had the strongest relationship among cohesion variables and competitive A-state variables.

The findings from the hierarchical multiple regression analyses showed that Atrait was a strong predictor of A-state, as expected. This finding confirms that competitive A-trait is the best predictor of A-state (Gill \& Martens, 1977; Martens et al., 1990; Smith et al., 1998). Furthermore, ATG-T contributed to the prediction of cognitive and somatic A-state beyond the variance explained by competitive A-trait. Again, competitive A-state was not predicted by any other dimensions of cohesion. That is, ATG-T is the most important cohesion component in the relationship between cohesion and competitive A-state.

ATG-T was the main cohesion attribute to influence competitive A-state in the 
present study and the findings are in line with previous research (Eys et al., 2003; Prapavessis \& Carron, 1996). One possible reason for this can be found from participants' soccer background information. Primary motivation to participate on the current team was apparently pertinent to task-related characteristics (e.g., enjoying the game and participation in competition). ATG-T items such as "I do not like the style of play on this team" or "I'm not happy with the amount of playing time I get" reflect these motives among recreational soccer players. Perception of anxiety results from interpretation of a disparity between environmental demands and intrapersonal characteristics (Lazarus, 1991; Martens et al., 1990). It is probable that when high task cohesion (ATG-T) is present, intrinsic motivation for participation in sport competition may be satisfied, and thus, little anxiety is perceived by recreational soccer participants. On the other hand, when this motivation is not met (e.g., few opportunities to play), negative feelings may increase. Another possible explanation for this finding is that psychological costs (e.g., psychological stress experienced from sport teams) may be diminished when individuals hold high levels of task cohesion (Prapavessis \& Carron, 1996). Specifically, the perceived pressure of responsibility may be reduced in highly task cohesive sport teams. Further, Eys et al. (2002) viewed "the pressure to carry out responsibilities and satisfy the expectations of others as task oriented activities" (p. 68).

In contrast to the hypothesis, GI-T was not a significant predictor of competitive Astate. It is difficult to understand why competitive A-state was not predicted by GI-T. One possible explanation is that GIT was highly correlated with ATG-T and moderately correlated with competitive Atrait. Possibly, shared variance of GI-T with ATG-T prevented the significant contribution of GI-T to the regression model. Also, it may be that the GI-T scale is not very accurate for recreational soccer players. For instance, Cronbach's Alpha of GI-T was fairly low $(\alpha=.64)$. In particular, participants had divergent responses to some items about practice. Because it was found that the majority of participants have little or no practice, these items might not be pertinent. Along with little or no weekly team practice, reconstitution of teams in every competition may have affected GI-T; team membership was relatively stable with some teams whereas turnover was higher on other teams. The wide range of years spent on the current team in both Study 1 (range: 0 to $16 ; M=4.68 ; S D=4.06)$ and Study 2 (range: 0 to $22 ; M=3.8 ; S D=4.05$ ) could have masked the results. Carron et al. (1998) stated that team members who stick to the group for a long time may be more receptive to group involvement than newcomers. However, it should be noted that constitution and reconstitution of teams in recreational soccer competitions are common. Future research may aim for a 
larger sample, which allows for controlling for years on the current team.

As expected, social cohesion did not contribute to the relationship between cohesion and competitive A-state. That is, social cohesion does not increase nor decrease competitive A-state. This result is in keeping with Prapavessis and Carron's (1996) findings of no relationship between social aspects of cohesion and competitive A-state variables. One possible explanation for this may be that social cohesion is an irrelevant factor to conditions that elicit stress. In the case of social cohesion, most recreational soccer players did not participate in the competition for the social purposes and may not perceive social cohesion as relevant to A-state responses. In support of this, only approximately $10.2 \%$ of the participants reported that they play for the current team to socialize.

Another possible explanation may be due to an indirect link of social cohesion with sport competition. For instance, GI-S items do not imply any sport competition context (e.g., our team members rarely party together), while ATG-T items have more direct relevance for sport competition (e.g., I'm not happy with the amount of playing time I get). Therefore, the context of items might impact the extent to which cohesion and competitive A-state are related.

In spite of the findings demonstrating the significant association between task cohesion and competitive A-state as well as the contribution of ATG-T in predicting competitive A-state, this study has several limitations. First, most participants were male (89.8\%) with only $10.2 \%$ female participants in Study 2. However, it should be noted that no significant difference in cohesion and anxiety was found between male and female participants. When correlation, stepwise and hierarchical multiple regression analyses were performed without female participants, the same results were found; ATG-T was the main predictor of competitive A-state and no other cohesion variables added a unique contribution to the prediction.

Second, there were some potential confounding variables for the relationship of cohesion to competitive anxiety. Specifically, experience and age ranges were wide in the current sample, and both have been found to be correlated to competitive A-state (Gould et al., 1984). Older and more experienced participants may have less competitive A-state at recreational competitive level. Even though participants noted a fairly high level of importance of the competition, the level of importance for recreational sports is different from professional sports and may have differently affected the level of anxiety experienced by participants. This could have affected the findings of this study. Sample size did not allow for control of age and experience in the current research, but it is suggested that experience and age be controlled in future studies. Although this study renders better understanding of a specific sport group and the sample used in this study was purposefully chosen, the findings of this 
study might not generalize to populations with different characteristics, such as age, skill level, experience and gender. Such personal attributes might alter cohesionanxiety relationships.

Third, some dimensions of cohesion had fairly low reliability. In particular, ATGS (Study 1, $\alpha=.37$; Study 2, $\alpha=.32$ ) was lower than acceptable and so removed from further analyses. GI-S (Study 1, $\alpha=.59$; Study $2, \alpha=.67$ ) was low, but kept for further analyses as it was close to acceptable reliability. It should be noted that the Cronbach's Alpha results in the present research were similar to those reported by Prapavessis and Carron (1996). Cronbach's Alpha of ATG-S $(\alpha=.40)$ was lower than adequate and removed from further analyses in Prapavessis and Carron's (1996) study. Salminen and Luhtanen (1998) also reported low internal consistency values. But, as Carron et al. (2002) pointed out, low reliabilities on some dimensions of cohesion are not all unexpected flukes because cohesion is a dynamic multifaceted construct. Nunnally (1978) suggested that acceptable reliability is around $\alpha=.70$ and the Cronbach's Alpha values in our study are close to $\alpha=.70$.

Fourth, it is obvious that the present study cannot infer any causal relationship between cohesion and competitive A-state. Despite the clear association between cohesion and competitive A-state, the research did not address how cohesion variables impact subcomponents of competitive A-state. Therefore, future research should employ experimental designs to examine whether manipulated team cohesion influences subsequent levels of competitive A-state. For instance, it is possible that a cohesion intervention could be used to lower competitive A-state, and in turn, foster continued participation in sport. Moreover, longitudinal designs may help researchers find out potential mechanisms for the effects of cohesion on individual anxiety.

Future research may further explore the complex relationships of cohesion and a wider range of affect states. Terry et al. (2000) found a positive association between cohesion and vigor and a negative association between cohesion and depression, anger, and tension. Also, it has been proposed by some researchers that anxiety may include a wide spectrum of affective states with some potentially positive aspects of anxiety (Cheng \& Hardy, 2016; Cheng, Hardy, \& Markland, 2009). Finally, Eys et al. (2003) found that high task cohesion is associated with positive interpretation of pre-competition symptoms.

Conducing follow-up interviews in future research may provide more in-depth understanding of the role of cohesion in individual anxiety among recreational athletes. Some researchers have used qualitative methods to advance understanding of complex phenomenon of anxiety and stress in the context of competition (Mellalieu, Neil, Hanton \& Fletcher, 2009; Neil, Mellalieu, \& Hanton, 
2009). Given that the features of cohesion and anxiety that are ever-evolving and dynamic make it difficult to study, observing processes of athlete's experience may contribute to fuller understanding of cohesion-anxiety relationships.

Despite the limitations, the findings from this study have important implications. Cohesion was related to competitive state anxiety beyond the prediction of competitive trait anxiety. The results of this study show that task cohesion has a negative relationship with competitive Astate in recreational sport level participants. Additionally, ATG-T predicted a significant proportion of competitive A-state beyond the contribution of competitive A-trait, indicating that ATG-T was the only relevant variable to competitive $A$-state at a recreational competition level. The findings of this study suggest that amateur soccer players who perceive high ATG-T experience lower competitive A-state. Additionally, the current findings extend our understanding of the relationship between cohesion and competitive anxiety beyond elite athletes to recreational athletes. Most recreational soccer athletes participate for fun, competition, and social interaction. It is likely that individual goals are satisfied with high perception of ATG-T, which may reduce competitive A-state.

The findings provide an important stepping stone for research on recreational athletes. Research on cohesion and anxiety has often pertained to performance. Given the larger number of people engaging in recreational team sports over the past few years opposed to slightly decreasing number of participants in individual sports (Physical Activity Council, 2016), more attention should be placed on individual experiences in recreational team sports. Cohesion has been found to be associated with adherence behavior in sport (Spink \& Carron, 1993). It has also been found that negative affect leads to drop out or burnout (Smith, 1986). Thus, if competitive anxiety diminishes fun, which in turn results in dropout, then cohesion may help recreational athletes have fun and stick to sports. Future research on the relationship of cohesion interventions to competitive A-state may help clarify models and potential mechanisms for the correlation of cohesion with precompetition anxiety responses, and thus enhance our understanding of group dynamics in sport. 


\section{References}

Baumeister, R.F., \& Leary, M.R. (1995). The need to belong: desire for interpersonal attachments as a fundamental human motivation. Psychological Bulletin, 117, 497-529. http://dx.doi.org/10.1037/00332909.117.3.497

Beauchamp, M. R., Bray, S. R., Eys, M. A., \& Carron, A. V. (2003). The effect of role ambiguity on competitive state anxiety. Journal of Sport and Exercise Psychology, 25, 77-92. http://dx.doi.org/10.1123/jsep.25.1. 77

Bosselut, G., Heuzé, J. P., Eys, M. A., \& Bouthier, D. (2010). Influence of task cohesion and role ambiguity on cognitive anxiety during a European rugby union championship. Athletic Insight, 2, 17-34.

Brawley, L.R., Carron, A.V., \& Widmeyer, W.N. (1987). Assessing the cohesion of teams: Validity of the group environment questionnaire. Journal of Sport Psychology, 9, 275-294. http://dx.doi.org/10.1123/jsp.9.3.27 5

Carron, A.V., Brawley, L.R., \& Widmeyer, W.N. (1998). The measurement of cohesiveness in sport groups. In J. L. Duda (Ed.), Advances in sport and exercise psychology measurement (pp. 213226). Morgantown, WV: Fitness Information Technology.

Carron, A.V., Brawley, L.R., \& Widmeyer, W.N. (2002). The group environment questionnaire test manual. Morgantown, WV: Fitness Information Technology.

Carron, A.V., Colman, M.M., Wheeler J., \& Stevens, C. (2002). Cohesion and performance in sport: A meta analysis. Journal of Sport and ExercisePsychology, 24, 168-188. http://dx.doi.org/10.1123/jsep.24.2. 168

Carron, A.V., Shapcott, K.M., \& Burke, S.M. (2007). Group cohesion in sport and exercise: Past, present and future. In M.R. Beauchamp \& M.A. Eys (Eds.), Group dynamics in exercise and sport psychology: Contemporary themes (pp. 117-139). New York: Routledge.

Carron, A. V., Widmeyer, W.N., \& Brawley, L.R. (1985). The development of an instrument to assess cohesion in sport teams: The group environment questionnaire. Journal of Sport Psychology, 7, 244-266. http://dx.doi.org/10.1123/jsp.7.3.24 4

Cheng, W. N. K., \& Hardy, L. (2016). Three-dimensional model of performance anxiety: Tests of the adaptive potential of the regulatory dimension of anxiety. Psychology of Sport and Exercise, 22, 255-263. http://dx.doi.org/10.1016/j.psychsp ort.2015.07.006

Cheng, W. N. K., Hardy, L., \& Markland, D. (2009). Toward a threedimensionalconceptualization of performance anxiety: Rationale and 
initial measurement

development. Psychology of Sport and

Exercise, 10, 271-278.

http://dx.doi.org/10.1080/17509840

701827445

De Francesco, C., \& Burke, K. L. (1997).

Performance enhancement strategies

used in a professional tennis

tournament. International Journal of

Sport Psychology, 28, 185-195.

Eys, M.A., Hardy, J., Carron, A.V., \&

Beauchamp, M.R. (2003). The

relationship between task cohesion and competitive state anxiety. Journal of Sport and Exercise Psychology, 25, 66-

76.http://psycnet.apa.org/doi/10.10 37/e548052012-729

Gill, D. L., \& Martens, R. (1977). The role of task type and success-failure in group competition. International Journal of Sport Psychology, 8, 160-177.

Gould, D., Petlichkoff, L., \& Weinberg, R.S. (1984). Antecedents of, temporal changes in, and relationships between CSAI-2 subcomponents. Journal of Sport Psychology, 6, 289-304. http://dx.doi.org/10.1123/jsp.6.3.28 9

Hanin, Y. L. (2007). Emotions and athletic performance: Individual zones of optimal functioning model. In D. Smith \& M. Bar-Eli (Eds.), Essential readings in sport and exercise psychology (pp. 55-73). Champaign, IL: Human Kinetics.

Hanton, S., Neil, R. and Mellalieu, S.D. (200 8). Recent developments in competitive anxiety direction and competition stress research. International Review of Sport and Exercise Psychology, 1, 4557. http://dx.doi.org/10.1080/17509 840701827445

Jones, J.G., \& Swain, A. (1992). Intensity and direction as dimensions of competitive state anxiety and relationships with competitiveness. Perceptual and Motor Differences, 17, 657663.

http://dx.doi.org/10.2466/pms.74.2. 467-472

Jones, M. V., \& Uphill, M. (2004).

Responses to the competitive state anxiety inventory-2 (d) by athletes in anxious and excited scenarios. Psychology of Sport and Exercise, 5, 201-212. http://dx.doi.org/10.1016/S14690292(02)00054-7

Landers, D. M., \& Arent, S. M. (2010). Arousal-performance relationships. In J. M. Williams (Ed.), Applied sport psychology: Personal growth to peak. performance (pp. 221-246). New York: McGraw-Hill Education.

Lazarus, R. (1991). Emotion and adaptation. New York: Oxford University Press.

Levine, L. J. (1996). The anatomy of disappointment: A naturalistic test of appraisal models of sadness, anger, and hope. Cognition and Emotion, 10, 337-360.

http://dx.doi.org/10.1080/02699939 6380178 
Lowther, J., \& Lane, A. (2002).

Relationships between mood, cohesion and satisfaction with performance among soccer players. Atbletic Insight, 4, 57-69.

Martens, R. (1977). Sport competition anxiety test. Champaign, IL: Human Kinetics. Martens, R., Vealey, R.S., \& Burton, D. (1990). Competitive anxiety in sport. Champaign, IL: Human Kinetics. Mellalieu, S. D., Neil, R., Hanton, S., \& Fletcher, D. (2009). Competition stress in sport performers: Stressors experienced in the competition environment. Journal of Sports

Sciences, 27, 729-744. http://dx.doi.org/10.1080/02640410 902889834

Neil, R., Mellalieu., S. D., \& Hanton, S. (2009). The contribution of qualitative inquiry towards understanding competitive anxiety and competition stress. Qualitative Research in Sport and Exercise, 1, 191205.

http://dx.doi.org/10.1080/19398440 902909058

Ntoumanis, N., \& Biddle, S. J. (2000).

Relationship of intensity and direction of competitive anxiety with coping strategies. The Sport psychologist, 14, 360-371. http://dx.doi.org/10.1123/tsp.14.4.3 60

Nunnally, J. C. (1978). Psychometric theory.

New York: McGraw-Hill.
Ostrow, A.C., \& Ziegler, S.G. (1978). Psychometric properties of the sport competition anxiety test. In B. Kerr (Ed.), Human performance and behavior (pp. 139-142). Calgary, AB: University of Calgary.

Paradis, K. F., Martin, L. J., \& Carron, A. V. (2012). Examining the relationship between passion and perceptions of cohesion in athletes. Sport and Exercise Psychology Review, 8, 22-31.

Patterson, M. M., Carron, A. V., \& Loughead, T. M. (2005). The influence of team norms on the cohesion-self-reported performance relationship: A multi-level analysis. Psychology of Sport and Exercise, 6, 479493. DOI:

10.1016/j.psychsport.2004.04.004

Physical Activity Council. (2016). 2016 Participation report: The physical activitycouncil's annual study tracking sports, fitness, and recreation participation in the U.S. http://www.physicalactivitycouncil.c om/PDFs/current.pdf

Prapavessis, H., \& Carron, A.V. (1996). The effect of group cohesion on competitive state anxiety. Journal of Sport and Exercise Psychology, 18, 64-74. http://dx.doi.org/10.1123/jsep.18.1. 64

Smith, R. E. (1986). Toward a cognitiveaffective model of athletic burnout. Journal of Sport Psychology, 8, 36-50. http://dx.doi.org/10.1123/jsp.8.1.36 
Smith, R.E., Smoll, F.L., \& Wiechman, S.A. (1998). Measuring trait anxiety in sport. In J.L. Duda (Ed.), Advances in sport and exercise psychology measurement (pp. 105-127). Morgantown, WV: Fitness Information Technology.

Spielberger, C.D. (1966). Anxiety and behavior. New York: Academic Press.

Spink, K. S., \& Carron, A. V. (1993). The effects of team building on the adherence patterns of female exercise participants. Journal of Sport and Exercise Psychology, 15, 39-49. http://dx.doi.org/10.1123/jsep.15.1. 39

Spink, K. S., Nickel, D., Wilson, K., \& Odnokon, P. (2005). Using a multilevel approach to examine the relationship between task cohesion and team task satisfaction in elite ice hockey players. Small Group Research, 36, 539-554.

http://dx.doi.org/10.1177/10464964 05275229

Terry, P.C., Carron, A.V., Pink, M.J., Lane, A.M., Jones, G.J., \& Hall, M.P. (2000). Perceptions of group cohesion and mood in sport teams. Group Dynamics: Theory, Research, and Practice, 4, 244-253. http://dx.doi.org/10.1037/10892699.4.3.244

Weinberg, R. S., \& Gould, D. (2014). Foundations of sport and exercise psychology, Champaign, IL: Human Kinetics.
Widmeyer, W. N., Brawley, L. R., \& Carron, A. V. (1990). The effects of group size in sport. Journal of Sport and Exercise Psychology, 12, 177-190. http://dx.doi.org/10.1123/jsep.12.2. 177 


\section{Tables and Appendix}

Table 1

Means, Standard Deviation, and Reliability Results for All Variables

\begin{tabular}{|c|c|c|c|c|}
\hline & \multicolumn{2}{|c|}{ Study 1} & \multicolumn{2}{|c|}{ Study 2} \\
\hline & $M(S D)$ & Reliability & $M(S D)$ & Reliability \\
\hline ATG-T & $28.30(6.91)$ & .76 & $29.88(6.20)$ & .76 \\
\hline ATG-S & $22.72(5.52)$ & .37 & $28.22(6.22)$ & .32 \\
\hline GI-T & $25.02(5.42)$ & .62 & $31.44(6.72)$ & .64 \\
\hline GI-S & $23.28(5.79)$ & .59 & $21.43(6.50)$ & .67 \\
\hline Cognitive A-state & $17.26(5.64)$ & .87 & $16.56(5.51)$ & .89 \\
\hline Somatic A-state & $16.19(5.20)$ & .85 & $15.75(5.79)$ & .92 \\
\hline A-trait & $18.51(4.54)$ & .82 & $17.20(4.69)$ & .79 \\
\hline
\end{tabular}


Table 2

Pearson Correlation Results for All Variables

\begin{tabular}{lccccccc}
\hline & ATG- & ATG-S & GI-T & GI-S & Cognitive & Somatic & A-trait \\
& T & & & & A-state & A-state & \\
& & & & & & & \\
\hline ATG-T & - & $.43^{* *}$ & $.57^{* *}$ & $.46^{* *}$ & -.28 & $-.37^{* *}$ & .09 \\
ATG-S & $.36^{* *}$ & - & $.35^{*}$ & $.51^{* *}$ & -.12 & -.08 & .20 \\
GI-T & $.56^{* *}$ & $.38^{* *}$ & - & $.48^{* *}$ & -.09 & -.12 & .27 \\
GI-S & $.22^{*}$ & $.55^{* *}$ & $.34^{* *}$ & - & -.27 & $-.32^{*}$ & .10 \\
Cognitive A-state & $-.35^{* *}$ & -.10 & $-.23^{*}$ & -.05 & - & $.71^{* *}$ & $.32^{*}$ \\
Somatic A-state & $-.29^{* *}$ & -.08 & $-.24^{*}$ & -.05 & $.72^{* *}$ & - & $.41^{*}$ \\
A-trait & -.17 & -.07 & $-.24^{*}$ & -.01 & $.61^{* *}$ & $.77^{* *}$ & - \\
\hline
\end{tabular}

**. Correlation is significant at the .01 level (2-tailed).

*. Correlation is significant at the .05 level (2-tailed).

Study 1: above diagonal/ study 2: below diagonal

Journal of Amateur Sport Volume Three, Issue Two

Oh, 2017 
Table 3

Hierarchical Multiple Regression Results for SCAT, GEQ-CSAI - Study 1

\section{Cognitive A-state regression model}

Step1: A-trait only

Model: $R=.32, F(1,45)=5.28^{*}$

Predictor: A-trait: $\beta=.32, t=2.30^{*}$

Step 2: A-trait + ATG-T, GI-T and GI-S

Model: $R=.45, F(2,44)=5.51^{* *}$

Predictor: A-trait: $\beta=.35, t=2.61^{*}$

Predictor: ATG-T: $\beta=-.31, t=-2.29^{*}$

\section{Somatic A-state regression model}

Step1: A-trait only

Model: $R=.41, F(1,45)=9.14^{* *}$

Predictor: A-trait: $\beta=.41, t=3.02^{* *}$

Step 2: A-trait + ATG-T, GI-T and GI-S

Model: $R=.58, F(2,44)=10.98^{* * *}$

Predictor: A-trait: $\beta=.45, t=3.63^{* * *}$

Predictor: ATG-T: $\beta=-.41, t=-3.29^{* *}$

Predictor variables: A-trait, ATG-T, GI-T and GI-S

${ }^{*} p<.05$. 
${ }^{* *} p<.01$.

${ }^{* * *} p<.001$.

Table 4

Hierarchical Multiple Regression Results for SCAT, GEQ-CSAI - Study 2

\section{Cognitive A-state regression model}

Step1: A-trait only

Model: $R=.61, F(1,86)=51.05^{* * *}$

Predictor: A-trait: $\beta=.61, t=7.15^{* * *}$

Step 2: A-trait + ATG-T, GI-T and GI-S

Model: $R=.66, F(2,85)=32.85^{* * *}$

Predictor: A-trait: $\beta=.57, t=6.86^{* * *}$

Predictor: ATG-T: $\beta=-.26, t=-3.09^{* *}$

\section{Somatic A-state regression model}

Step1: A-trait only

Model: $R=.77, F(1,86)=121.72^{* * *}$

Predictor: A-trait: $\beta=.77, t=11.03^{* * *}$

Step 2: A-trait + ATG-T, GI-T and GI-S

Model: $R=.78, F(2,85)=67.05^{* * *}$

Predictor: A-trait: $\beta=.74, t=10.76^{* * *}$

Predictor: ATG-T: $\beta=-.16, t=-2.39^{*}$ 


\section{Predictor variables: ATG-T, GI-T and GI-S}

$$
\begin{aligned}
& { }^{*} p<.05 . \\
& { }^{* *} p<.01 . \\
& { }^{* * *} p<.001 .
\end{aligned}
$$




\section{Appendix}

Modified Competitive State Anxiety Inventory-2 (CSAI-2)

Instructions: Please read the scenario below. Use this scenario and try to put yourself into the situation to answer the following questions.

\section{Soccer competition scenario}

Now imagine you and your teammates are getting ready for the most important competition. You look around the field and see that some of your teammates are wearing soccer cleats while others are passing the ball around. The opponent team players are warming up at the opposite half of the field. While you are warming up, one of your teammates asks "Are you ready for the game?" The referees are walking to the center circle and the opponent team is gathering to the center of the field. The game is now about to start. Think about how you feel in that situation, right at

\section{that moment.}

Instructions: A number of statements that athlete use to describe their feelings before competition are given below. Read each statement and then circle the appropriate number to indicate how you feel in the above situation right at that moment, in the most important game. There are no right or wrong answers. Do not spend too much time on any one statement, but choose the answer that best describes how you feel right at that moment. 